Supporting information for

\title{
LAN: A Materials Notation for 2D Layered Assemblies
}

\author{
Georgios A. Tritsaris, ${ }^{\dagger}$ Yiqi Xie, ${ }^{\ddagger}$ Alexander M. Rush, ${ }^{\dagger}, \S$ Stephen Carr, \\ Marios Mattheakis, ${ }^{\dagger}$ and Efthimios Kaxiras ${ }^{\dagger}$, \\ ${ }^{\dagger}$ John A. Paulson School of Engineering and Applied Sciences, Harvard University, \\ Cambridge, Massachusetts 02138, USA \\ ¥Institute for Applied Computational Science, Harvard University, Cambridge, \\ Massachusetts 02138, USA \\ "Physics Department, Harvard University, Cambridge, Massachusetts 02138, USA \\ $\S$ Current address: Cornell Tech, New York, NY, USA
}

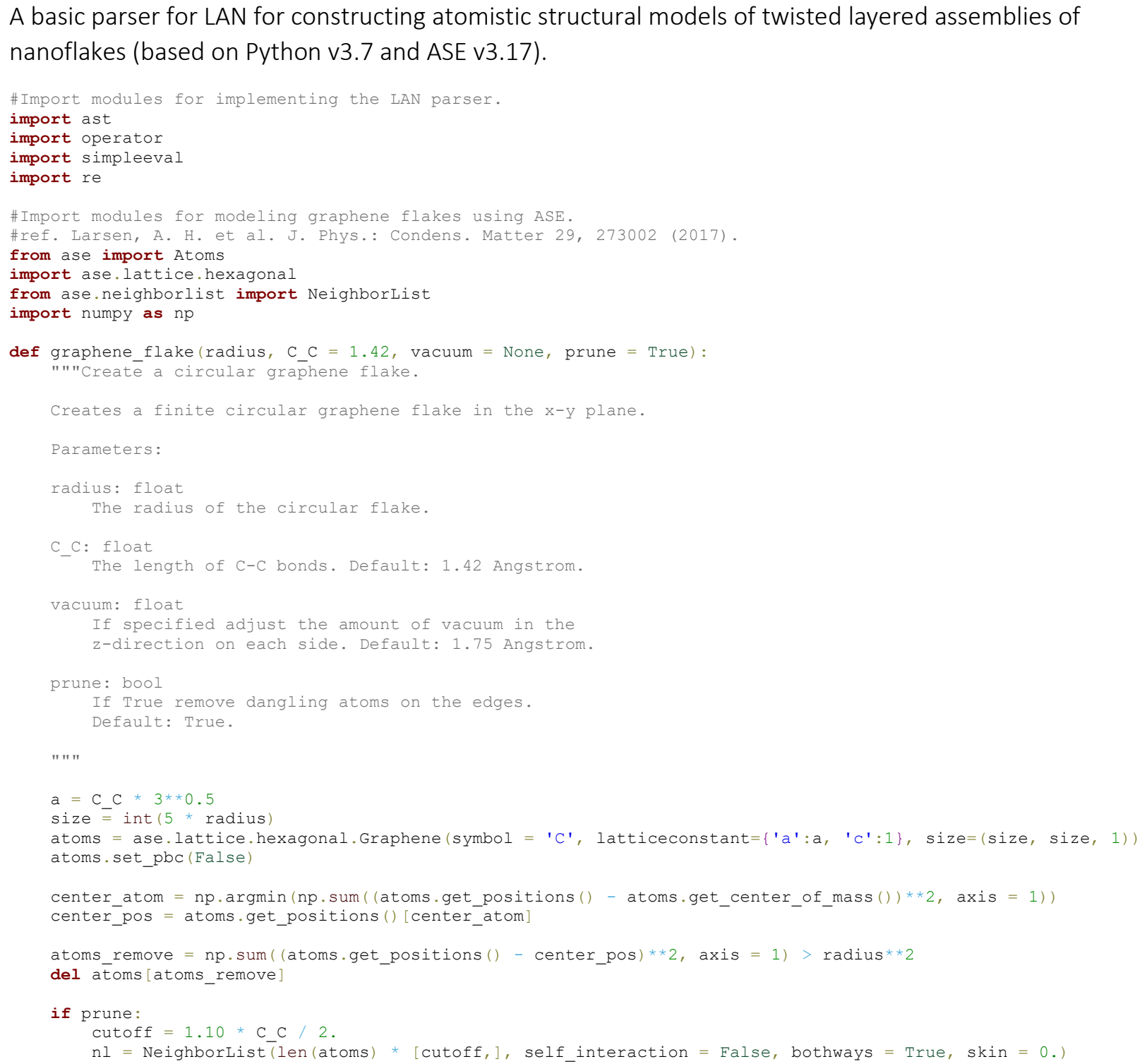




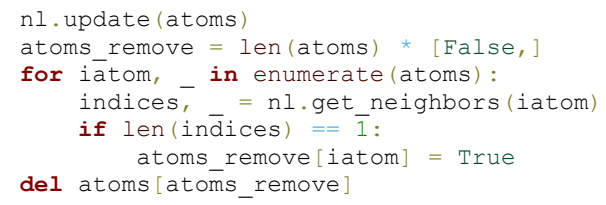

return atoms

def layered assembly (notation, blocks $=$ None, vacuum $=$ None) : "" Create a layered assembly.

Creates a layered assembly in the $z$-direction.

Parameters:

notation: string

The string-based notation describing the layered assembly.

For example, the string G/G@1.08 describes a bilayer

with twist angle of 1.08 degrees. The symbols / and a are used to describe the binary operations of the vertical stacking of a layer or layered (sub)structure on another, and counterclockwise rotation of a layer or layered

(sub)structure by some angle about the stacking direction (in degrees; assuming the same absolute coordinate system), respectively. The grammar is described in detail in the manuscript. 


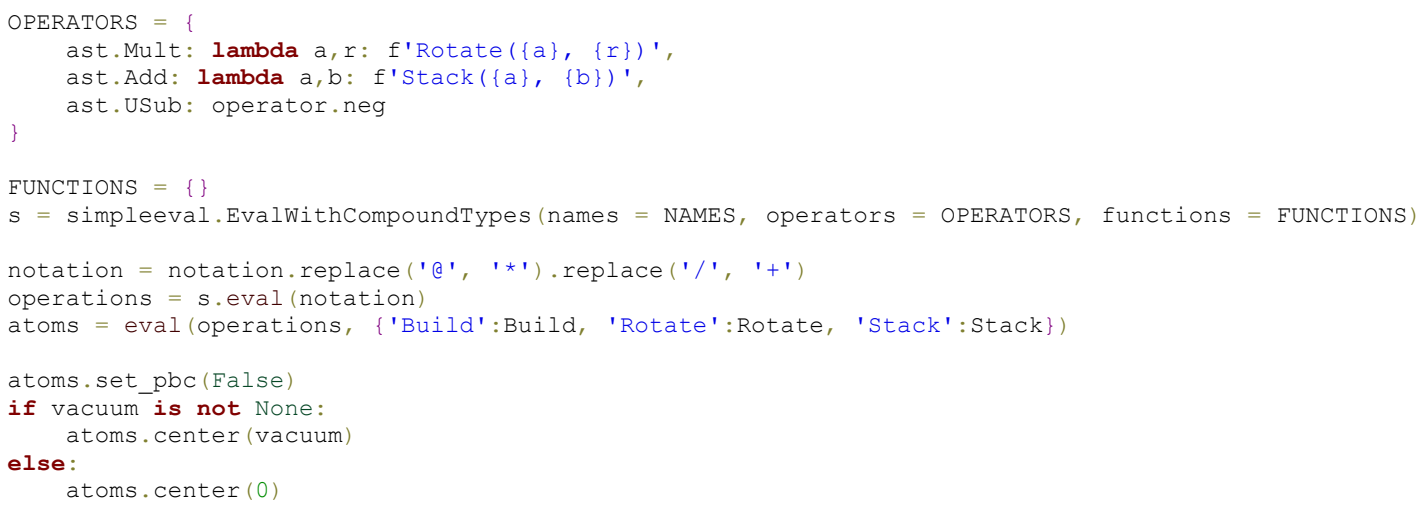

return atoms

\#\#\#\# Example of using LAN to build

\#\#\#\# atomistic models of arbitrarily layered assemblies

\#\#\#\# of finite graphene flakes using ASE.

from ase.visualize import view

for twist in $[0.5,5,10,20]$ :

\#Build a twisted double bilayer of graphene flakes.

atoms = layered assembly $\left('(G / G) /(G / G)\right.$ a $\%$ ' $\frac{\circ}{0}$ twist, vacuum $=5$.)

view (atoms) 